\title{
Triatoma infestans em área sob vigilância entomológica para doença de Chagas, Estado de São Paulo, Brasil
}

\author{
Triatoma infestans in area under entomological surveillance for \\ Chagas' disease in São Paulo State, Brazil
}

\author{
Odair F. Leite ${ }^{1}$, Maria José C.P. Alves', Savina S.L. Souza1, Renata C. Mayo', \\ Valmir R. Andrade ${ }^{1}$, Celso E. Souza ${ }^{1}$, Osias Rangel ${ }^{1}$, Susely S. Oliveira ${ }^{1}$, \\ Virgilia L.C. Lima', Vera L.C.C. Rodrigues', Maria Esther de Carvalho', \\ Claudio Casanova' ${ }^{1}$ e Dalva M.V. Wanderley ${ }^{1}$
}

\begin{abstract}
Resumo Atendendo a notificação de encontro, por habitantes da Fazenda Paraíso, área rural do Município de Paulínia, Estado de São Paulo, de um exemplar alado de triatomíneo, procedeu-se a uma investigação epidemiológica em que capturaram-se 109 exemplares de Triatoma infestans em focos situados em construçôes peridomiciliares. As condições locais favoreciam a colonização por triatomíneos: grande número de construções peridomiciliares abandonadas, habitadas por pombos e pardais, fornecedores de farto alimento para os hemípteros. Eliminaram-se os focos por meio de controle mecânico dos ninhos dos pássaros. Borrifaram-se, com inseticida de ação residual, todas as unidades domiciliares da região. Desde que persistam as condições para a instalação de focos de triatomíneos nessa localidade, é obrigatória a implementação de ações de vigilância. Entretanto, os indicadores entomológicos e sorológicos sugerem não ser preocupante a situação atual. Destaca-se aqui a importância da notificação triatomínica para a detecção de focos de triatomíneos, particularmente os de Triatoma infestans.
\end{abstract}

Palavras-chaves: Triatoma infestans. Doença de Chagas. Vigilância entomológica.

\begin{abstract}
In response to notification of the capture of a winged triatomine bug by local inhabitants, an epidemiological investigation was investigated in the rural area of the Municipality of Paulínia, São Paulo State (Brazil). This led to the collection of 109 specimens of Triatoma infestans from peridomiciliary breeding sites. Local conditions favored colonization by triatomine bugs: many abandoned outbuildings were inhabited by pigeons and sparrows, which meant abundant feed for these bugs. Thus, surveillance is mandatory as long as conditions for triatomine breeding persist. Nevertheless, entomological and serological indicators ruled out immediate concern over vectorial transmission of Chagas' disease in this municipality. All domiciliary units of the region were sprayed with residual-effect insecticides and the triatomine breeding sites were destroyed by the use of mechanical control of bird nests. The importance of reporting the occurrence of triatomine bugs, particularly Triatoma infestans, in this region, is emphasized.
\end{abstract}

Key-words: Triatoma infestans. Chagas' disease. Entomological surveillance.

Iniciado em 1951, o Programa de Controle da Doença de Chagas no Estado de São Paulo priorizou, a partir de 1964, o combate químico integral ao Triatoma infestans, restrito a ecótopos artificiais. Esse controle se estendeu por toda a região endêmica, representada por uma área ampla e contínua, compreendendo todo o planalto paulista e incluindo 231 municípios dos 369 então existentes ${ }^{8}$. Introduzido a partir da Região Sorocabana, Triatoma infestans se dispersou, ao longo do Estado, em casas onde as condições favoreciam a colonização ${ }^{13}$. Como consequeência do controle instituído no final dos anos 1960, esta espécie começou a desaparecer das áreas trabalhadas, não ocorrendo o mesmo com Triatoma sordida e Panstrogylus megistus ${ }^{1}$. Ao lado das ações do Programa, a conquista de condições mais adequadas de moradia no campo e o êxodo rural $^{10}{ }^{13}$ favoreceram amplamente a melhoria da situação epidemiológica da doença de Chagas.

No ano de 1985, o Programa de Controle da Doença de Chagas determinou a pesquisa triatomínica de rotina por localidade e não por município, unidade de pesquisa até então ${ }^{1820}$. Naquele ano apenas 29 localidades, situadas na Região de Sorocaba, foram priorizadas para pesquisa triatomínica, devido ao fato de ter sido assinalada aí, em pelo menos um dos três

1. Superintendência de Controle de Endemias - SUCEN, São Paulo SP, Brasil.

Endereço para correspondência: Dra. Dalva Marli Valério Wanderley. R. Paula Souza 166, 01027-000 São Paulo, SP, Brasil.

Tel: 5511 227-0622; Fax 5511 229-8292

e-mail: dalva@sucen.sp.gov.br

Recebido para publicação em 27/10/2000. 
anos imediatamente anteriores, a presença de $T$. infestans ${ }^{20}$. Este vetor passou, a partir de então, a ser exaustivamente investigado. Disto resultou o registro, em 1986, da presença de um exemplar adulto, macho, na Região de Campinas, Município de MogiGuaçu, colhido em uma fresta de parede de tijolos, rebocada, durante reforma. Havia entretanto sinais evidentes de tratar-se de inseto morto desde há muito.

Após terem sido negativas reiteradas pesquisas de T. infestans no meio rural paulista e demonstrada, por meio de reações sorológicas, a ausência de casos positivos em menores de 10 anos, o que é atribuível à interrupção da transmissão vetorial da doença de Chagas, mantiveram-se, em áreas de infestação residual, as ações de vigilância entomológica sobre as espécies secundárias e pesquisa de triatomíneos, participando os habitantes locais na detecção de insetos invasores de seus domicílios ${ }^{17}{ }^{19}$. A vigilância entomológica da doença de Chagas no Estado de São Paulo preconiza a busca ativa de triatomíneos em áreas de infestação residual e o estímulo à notificação de triatomíneos por parte da população, com cobertura em todo o Estado ${ }^{18}$. O atendimento a cada notificação inclui pesquisa minuciosa na unidade notificante e instalação de medidas de controle mecânico e químico, quando necessário ${ }^{7}$. Os moradores das várias regiões são orientados, pelas equipes de campo e Postos de Informação de Focos de Triatomíneos (PIFTs) instalados em escolas rurais, a notificar o encontro de triatomíneos. Em qualquer situação, os insetos capturados devem ser levados a Centros de Saúde e, a partir destes, à Superintendência de Controle de Endemias (SUCEN), onde são identificados e submetidos a exame de conteúdo intestinal para pesquisa de Trypanosoma cruzi.
No período de 1990 a 1998 foram pesquisadas, dentro das atividades de rotina do Programa de Controle da Doença de Chagas no Estado de São Paulo, 127.408 unidades domiciliares. A presença de triatomíneos foi notificada pelos moradores em 17.381 dessas pesquisas. Os percentuais de infestação obtidos na rotina e nos atendimentos a notificação foram, respectivamente, 1,5 e 6,2. No período foram capturados 77533 triatomíneos, dos quais T. sordida correspondia a $86,6 \%$ e $P$. megistus a 13,4\%. No mesmo período, constatou-se a ocorrência isolada e dispersa de apenas 3 exemplares de T. infestans. Todos foram notificados por moradores da Região de Campinas, nenhum infectado por $T$. cruzi. Os atendimentos às notificações dos dois primeiros exemplares, procedentes de localidades dos municípios de Tapiratiba e Sumaré resultaram negativos, o que sugere que tenham sido os mesmos trazidos passivamente pelos próprios residentes, ao regressar de viagens a outros estados ainda infestados ${ }^{10,20}$. O atendimento à notificacão do terceiro exemplar de triatomíneo, proveniente de área rural do município de Paulínia, resultou no encontro de uma colônia e captura de 109 exemplares de T. infestans.

O encontro deste último foco, após mais de uma década sem registro de infestação importante por T. infestans no Estado de São Paulo, foi motivo de preocupação das autoridades da área de Saúde Pública. Este trabalho tem como objetivos relatar este episódio; mostrar a importância da notificação na detecção de focos de triatomíneos, particularmente de $T$. infestans e descrever as medidas de controle integrado implementadas visando a eliminar o foco, evitar a dispersão dos insetos para outras localidades e manter a continuidade da interrupção da transmissão natural da doença de Chagas.

\section{MATERIAL E MÉTODOS}

Área de Trabalho. O município de Paulínia está localizado a nordeste do Estado de São Paulo (Latitude $22^{\circ}, 46^{\prime}$, Longitude $\left.47^{\circ}, 10^{\prime}\right)$. Dista $118 \mathrm{~km}$ da capital e possui área de $154 \mathrm{~km}^{2}$. O clima é subtropical, com verão quente e inverno brando, registrando temperatura média de $21,6^{\circ} \mathrm{C}$. Com 44.440 habitantes e taxa de urbanização de $87,7 \%$, Paulínia foi distrito do município de Campinas, integrante da área endêmica chagásica original. Emancipou-se em 1964. O município é servido por importantes rodovias e o entroncamento ferroviário de Campinas propicia a Paulínia acesso a todas as regiões do estado e do país (Figura 1).

Atividades de investigação. Foram implementadas medidas de investigação de focos de T. infestans de acordo com o preconizado pelo Programa de Controle ${ }^{18}$, que inclui pesquisa de infecção natural dos triatomíneos coletados $^{9}$, identificação da fonte alimentar utilizada por esses hemípteros por meio de reação de precipitação em tubo capilar $^{15}$, frente a uma bateria de anti-soros de ave, marsupial, roedor e homem. Foi realizada coleta de sangue da população residente e trabalhadora da fazenda, com vistas à pesquisa de anticorpos anti- $T$. cruzi, utilizando-se reações de imunofluorescência indireta (RIFI) quantitativa, conforme descrito em Solís-Acosta e cols ${ }^{16}$. Foram ainda aplicadas reações de ensaio imunoenzimático (ELISA) com "kit"comercial Wiener ${ }^{\circledR}$, Rosário, Argentina, seguindo-se as recomendações do fabricante, exceto no que se refere a uso de eluatos de sangue total, que foi adaptado no Laboratório de Soroepidemiologia da SUCEN para diluições 1:32. Os indivíduos sorologicamente positivos foram investigados, classificados quanto à procedência e em seguida encaminhados para acompanhamento, no setor saúde. 


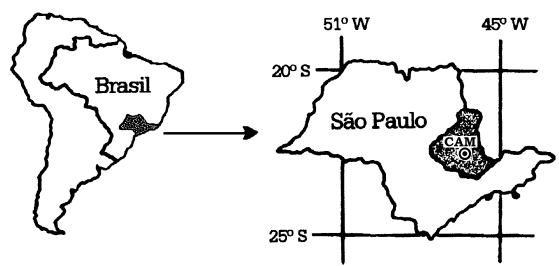

CAM -Região de Campinas

O Paulinia: sede do Governo Municipal

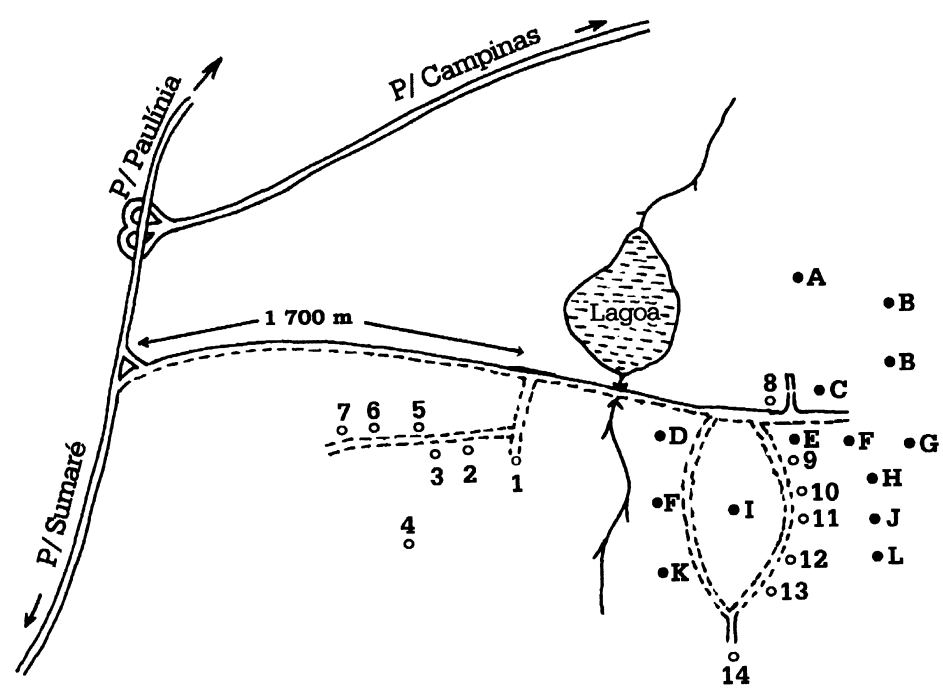

O Unidades domiciliares: 1 a 14

- A - Laticínios; B - Cocheira; C - Almoxarifado; D - Depósito de ração; E - Depósito de estruturas de arquibancadas; $\mathbf{F}$ - Depósito de aço desativado; G - Serralharia; H - Depósito de telhas; I - Barracão; J - Oficina mecânica; K - Usina de aguardente desativada; $\mathbf{L}$ - Sede da fazenda.

Figura 1 - Localizacão da área trabalhada na Fazenda Paraíso, Município de Paulínia, Estado de São Paulo, 1999.

Atividades de controle. A partir da notificação de encontro de exemplar de $T$. infestans, no município de Paulínia, Fazenda Paraíso, atualizou-se o reconhecimento geográfico desta região, tendo sido delimitada uma área de 1.500 metros ao redor do local de encontro. A pesquisa entomológica abrangeu as unidades domiciliares situadas em áreas rural e periurbana. Nas localidades realizaram-se atividades de controle físico, incluída aí a retirada de ninhos de pombos e pardais, queimados após minuciosa pesquisa triatomínica e controle químico do local. Devido à considerável altura das construções do peridomicílio da fazenda, contou-se com o auxílio de andaimes e de viatura do Corpo de Bombeiros dotada de auto-escada. Para a borrifação foi utilizado o piretróide Cipermetrina, na dosagem de $156 \mathrm{mg} / \mathrm{m}^{2}$, em todas as unidades domiciliares da Fazenda Paraíso.
No restante da zona rural pesquisada, estabeleceramse, como critérios de seleção das unidades domiciliares para borrifação, a distância entre estas e o foco e condições para o estabelecimento e proliferação de triatomíneos. $\mathrm{Na}$ área periurbana realizou-se somente pesquisa entomológica associada a orientação dos moradores para o encaminhamento, ao Posto de Saúde mais próximo, de quaisquer exemplares de insetos encontrados que julgassem ser barbeiros. Durante as pesquisas, 35\% das casas encontraram-se fechadas e/ou desabitadas. $\mathrm{Na}$ área periurbana foi dada orientação aos moradores e trabalhadores, com a utilização de folhetos educativos, mostruários de triatomíneos e cartilhas. Iniciaram-se os primeiros contatos para a instalação de Postos de Informação de Focos de Triatomíneos (PIFTs) nas escolas das localidades limítrofes. Tendo em vista 
que o peridomicílio, onde foi encontrado o maior número de exemplares de $T$. infestans, era utilizado como depósito de estruturas de arquibancadas para festas populares, foi solicitada pesquisa entomológica em todos os locais para onde este material se havia deslocado durante os anos de 1998 e 1999, perfazendo um total de 34 localidades de municípios paulistas e
7 mineiros, com o objetivo de verificar a possibilidade de dispersão passiva de $T$. infestans deste foco para outros municípios. Além das revisões de focos preconizadas pelo Programa de Controle de Doença de Chagas, os locais de encontro de T. infestans foram revistos mensalmente por um período de 6 meses.

\section{RESULTADOS}

No final de 1998, um morador da localidade notificou o encontro de um exemplar alado de T. infestans. $O$ atendimento a esta notificação não demonstrou a existência de outros exemplares do inseto. Estando a área sob vigilância, nova pesquisa foi realizada após um ano, constatando-se então a presença de duas ninfas e uma fêmea de T. infestans. A partir desta ocasião, desencadeou-se pesquisa minuciosa em toda a localidade, encontrando-se mais 106 exemplares dessa espécie (85 ninfas e 21 alados) em construções peridomiciliares. Dos 109 exemplares, 37 tiveram seus conteúdos intestinais examinados, não tendo sido demonstrada a presença de formas flageladas. Os demais estão sendo mantidos no insetário do Laboratório de Parasitoses por Flagelados da SUCEN, para outros estudos. A investigação da fonte alimentar utilizada por esses 37 exemplares apontou sangue de ave como principal alimento (Tabela 1).

Amostras de sangue de 57 indivíduos que moram ou trabalham na localidade, coletadas em papel-filtro, foram processadas pelas técnicas da imunofluorescência indireta e ELISA. Ambas as técnicas revelaram reação positiva em uma amostra correspondente a indivíduo com idade de 45 anos, sexo feminino que, tendo residido em área rural dos municípios de Carmo do Rio Claro e Alterosa, estado de Minas Gerais, transferiuse para o estado de São Paulo no início de 1999, onde residiu no município de Piracicaba por 6 meses e depois em Paulínia (Tabela 2).

Tabela 1 - Triatoma infestans na Fazenda Paraíso, Município de Paulínia, Estado de São Paulo, 1998-2000.

\begin{tabular}{|c|c|c|c|c|c|}
\hline \multirow{2}{*}{$\begin{array}{l}\text { Estágio } \\
\text { evolutivo }\end{array}$} & \multicolumn{2}{|c|}{ Captura } & \multirow{2}{*}{$\begin{array}{l}\text { Exemplares } \\
\text { examinados }\end{array}$} & \multirow{2}{*}{$\begin{array}{l}\text { Infecção por } \\
\text { T. cruzi }\end{array}$} & \multirow{2}{*}{ Fonte alimentar } \\
\hline & domiciliar & peridomiciliar & & & \\
\hline Alados & $2^{*}$ & 21 & 21 & negativo & ave \\
\hline Ninfas & - & 87 & 16 & negativo & ave \\
\hline Total & $2^{*}$ & 108 & 37 & - & - \\
\hline
\end{tabular}

* 1 exemplar notificado por morador.

Tabela 2 - Investigacão de infecção chagásica em moradores e funcionários da Fazenda Paraíso. Paulínia, Estado de São Paulo, 1999-2000.

\begin{tabular}{|c|c|c|c|c|c|c|}
\hline \multirow{2}{*}{$\begin{array}{l}\text { Faixa etária } \\
\text { (anos) }\end{array}$} & \multicolumn{4}{|c|}{ Amostras examinadas } & \multicolumn{2}{|c|}{ Amostras reagentes } \\
\hline & feminino & masculino & total & $\%$ & $\mathrm{n}^{\circ}$ & $\%$ \\
\hline$<05$ & 1 & 3 & 4 & 7,0 & 0 & 0,0 \\
\hline $6-9$ & 0 & 1 & 1 & 1,8 & 0 & 0,0 \\
\hline $10-14$ & 0 & 1 & 1 & 1,8 & 0 & 0,0 \\
\hline $15-19$ & 2 & 2 & 4 & 7,0 & 0 & 0,0 \\
\hline $20-29$ & 9 & 3 & 12 & 21,0 & 0 & 0,0 \\
\hline $30-39$ & 5 & 8 & 13 & 22,8 & 0 & 0,0 \\
\hline $40-49$ & 3 & 6 & 9 & 15,8 & 1 & 11,1 \\
\hline$\geq 50$ & 6 & 7 & 13 & 22,8 & 0 & 0,0 \\
\hline Total & 26 & 31 & 57 & 100,0 & 1 & 1,8 \\
\hline
\end{tabular}

$\mathrm{Na}$ área delimitada, 1.500 metros a partir do foco, foram pesquisadas 1.012 unidades domiciliares, 100 das quais na área rural e 912 em área periurbana. A borrifação atingiu 25 unidades domiciliares pertencentes à área rural, sendo 14 da Fazenda Paraíso e 11 pertencentes a outras localidades da área rural, segundo os critérios estabelecidos para a borrifação, conforme referido nas atividades de controle.

$\mathrm{Na}$ revisão de focos, realizada 30 dias após o controle químico (1 $1^{a}$ revisão), foram encontrados 2 exemplares de $T$. infestans no peridomicílio de uma unidade domiciliar da Fazenda Paraíso, sendo as demais negativas. O local de encontro foi um anexo 
(usina de aguardente desativada), onde se encontraram as maiores dificuldades para o controle químico. Devido a este fato, procedeu-se a nova borrifação, apenas neste anexo. Aos 60 dias após o primeiro controle químico, nova pesquisa foi realizada ( $2^{a}$ revisão), resultando negativa. Aos 6 meses do controle inicial a pesquisa permanecia negativa (Tabela 3).

Uma vez concluídas as atividades de controle, foi realizada avaliação das condições sanitárias da fazenda, com instruções ao proprietário sobre medidas de manejo do peridomicílio e interior dos anexos (limpeza, retirada de entulho, capinação, remoção de inservíveis como caixas de papelão e latas; telagem de tonéis para evitar criação de animais como ratos e gambás, acondicionamento adequado das ferragens e peças de carro, acondicionamento adequado dos grãos usados para alimentar o gado, telagem das janelas, remoção e destruição de sacaria de serragem, para evitar proliferação de lacraias). Foi observado que o alimento do gado, depositado no chão, constituise em atrativo para proliferação de pombos, sendo indicada a construção de cocho, coberto com tela, como forma de diminuir a oferta alimentar para os pombos.

Seis meses após o encontro deste foco não foi notificado, pelas ações de vigilância, nenhum outro achado deste vetor no estado de São Paulo. Pesquisas entomológicas nos locais para onde as estruturas de arquibancadas foram deslocadas encontram-se em desenvolvimento. No entanto, pesquisas já concluídas em municípios da região de Sorocaba, a última do estado a lograr a eliminação do T. infestans, resultaram negativas. Constatou-se que as arquibancadas eram montadas em áreas urbanizadas dos municípios, em grandes espaços abertos, o que significa pouco risco para a dispersão passiva de T. infestans do município de Paulínia para outros municípios.

Tabela 3 - Atividades de pesquisa triatomínica, controle e revisão de foco de Triatoma infestans. Paulínia, Estado de São Paulo, 1999-2000.

\begin{tabular}{|c|c|c|c|}
\hline \multirow{2}{*}{ Área } & \multicolumn{3}{|c|}{ Unidades domiciliares } \\
\hline & existentes & pesquisadas & borrifadas \\
\hline \multicolumn{4}{|l|}{ Controle Rural } \\
\hline Fazenda Paraíso & 14 & 14 & 14 \\
\hline Outras localidades & 86 & 79 & 11 \\
\hline Controle Periurbano & 912 & 594 & - \\
\hline Total & 1.012 & 687 & 25 \\
\hline \multicolumn{4}{|l|}{ Revisão } \\
\hline $1^{\underline{a}}$ Fazenda Paraíso & 14 & 14 & 1 \\
\hline 2ªzenda Paraíso & 14 & 14 & - \\
\hline
\end{tabular}

\section{DISCUSSÃO}

Embora a campanha contra T. infestans no Estado de São Paulo possa ser considerada como bem sucedida, as atividades de vigilância são mantidas, juntamente com o controle dirigido às demais espécies invasoras de ecótopos artificiais ${ }^{10}$. No contexto da Iniciativa do Cone Sul, a manutenção de vigilância em caráter permanente para a doença de Chagas, mesmo após a eliminação de T. infestans, foi destacada como indispensável para a consolidação dos resultados ${ }^{46}$. O Estado de São Paulo, que alcançou a meta de eliminação de $T$. infestans dos domicílios, conforme conclusões da Comissão Internacional de Avaliação ${ }^{4}$, realizada em novembro de 1995, apresenta hoje um espaço bastante reduzido de concentração triatomínica, com predomínio de $T$. sordida e P. megistus no ambiente domiciliar, restrito às poucas localidades nas quais têm sido implementadas pesquisas rotineiras casa-a-casa. No entanto, o serviço de controle tem olhado com prioridade absoluta para o atendimento às notificações de triatomíneos encaminhadas pela população, uma vez que esta atividade têm-se mostrado a mais eficaz na detecção de focos desses vetores nas análises recentemente realizadas ${ }^{5} 141820$. Na última década tem-se mantido continuidade das notificações, em toda a área de distribuição triatomínica, com a conseqüente visita à unidade notificante por parte do serviço de controle, sendo atingido grau de cobertura adequado.

O mapeamento inicial da zona endêmica chagásica paulista incluiu a Região de Campinas, devido a presença aí de $T$. infestans. Uma vez alcançado o seu controle, o número de domicílios infestados reduziu-se significativamente e a infestação passou a se dar quase que exclusivamente por $P$. megistus ${ }^{5}$. Esta área, onde se encontra a mais intensa infestação por este vetor em relação ao restante do estado, situase em "hipotética zona limítrofe" entre dois tipos de comportamento das populações de P. megistus, correspondendo, ao norte, à região de populações domiciliadas e ao sul a região de populações silvestres ${ }^{3}$. A notificação triatomínica está condicionada à descoberta de formas adultas da 
espécie, récem-chegadas ao domicílio em conseqüência da dispersão da espécie.

A detecção desse foco pela atividade de atendimento a notificação, ao contrário da idéia de falha operacional ou da permanência de focos residuais da espécie na área, indica que o sistema de vigilância entomológica, da forma como o Programa a preconiza, priorizando o atendimento a notificação de insetos, tem sido conduzido com eficácia no Estado de São Paulo, atuando de maneira satisfatória, com a pronta tomada de medidas pertinentes quando da identificação de cada achado. Os dados complementares da investigação: ausência de infecção natural dos vetores, utilização de sangue de aves como a fonte de alimentação e o encontro de apenas um indivíduo com sorologia positiva, demonstraram não ter havido evidência de risco de transmissão da doença de Chagas nas localidades investigadas. Após minuciosa investigação epidemiológica, o caso reagente sorológico foi associado a infecção pretérita, adquirida em área endêmica no Estado de Minas Gerais. Esta experiência reforçou a ênfase dada pelo Programa de Controle à persistência e ao incremento das atividades educativas tendentes a manter alertada a população e orientada para a pesquisa de insetos semelhantes aos triatomíneos e instruída quanto à atitude a ser tomada. Constatou-se, também, que um foco ainda limitado como este, apesar das dificuldades decorrentes das características dos imóveis dessa localidade, foi passível de controle efetivo em curto espaço de tempo.

O fluxo constante de estruturas para montagem de arquibancadas deste para outros estados com focos residuais de $T$. infestans pode ter sido responsável pela introdução passiva do triatomíneo gerador da colônia, uma vez que os últimos registros de infestação por esse vetor da doença de Chagas em municípios da Região de Campinas datam do início da década de $80^{11}{ }^{12}$, época em que o Programa de Controle apresentava cobertura integral da área endêmica paulista, com pesquisas triatomínicas de rotina.

A localidade Fazenda Paraíso oferece condições ideais para o estabelecimento de colônias de triatomíneos, devido ao grande número de anexos abandonados, servindo de abrigo para pombos e pardais, abundante fonte alimentar para esses hemípteros, daí justificando a implementação das ações de vigilância.

O foco encontrado estava restrito ao peridomicílio da Fazenda Paraíso e, provavelmente pela dificuldade de acesso para pesquisa e controles físico e químico, a colônia não foi totalmente eliminada, tendo sido encontrados ainda, durante revisão do foco, exemplares de $T$. infestans. O controle foi completado em ações de seguimento. A estrutura estadual de operações de campo e laboratórios, presente em todo o estado, tem garantido cobertura e continuidade, fatores importantes no desempenho das acões de vigilância e controle dos vetores da doença de Chagas. No entanto, a raridade dos encontros de $T$. infestans determina a necessidade de que se desenvolvam estratégias e metodologias para a capacitação das equipes de campo responsáveis pela pesquisa e borrifação dos domicílios e do pessoal dos laboratórios, com vistas a garantir a qualidade das ações pertinentes.

\section{AGRADECIMENTOS}

Ao Dr. Claudio Santos Ferreira, do Instituto de Medicina Tropical de São Paulo, pela revisão crítica do manuscrito.

\section{REFERÊNCIAS BIBLIOGRÁFICAS}

1. Buralli GM. Estudo do controle dos triatomíneos domiciliados no Estado de São Paulo. Dissertação de Mestrado. Faculdade de Saúde Pública da Universidade de São Paulo. São Paulo, 1985.

2. Dias JCP. Vigilância epidemiológica contra o Triatoma infestans. Revista da Sociedade Brasileira de Medicina Tropical 26(supl.III):39-44, 1993.

3. Forattini OP, Rocha e Silva EO, Ferreira AO, Rabello EX, Santos JLF, Lima AR. Aspectos ecológicos da tripanossomíase americana. XI - Domiciliação de Panstrongylus megistus e potencial enzoótico. Revista de Saúde Pública 11:527-550, 1977.

4. Informe de La Misión de Evaluación de Las Actividades de Control/Eliminación de Triatoma infestans y corte de La Transmission Transfusional de Chagas. Estados de São Paulo e Minas Gerais. Iniciativa Cono Sur, 6-15 noviembre, 1995.
5. Lima VLC, Yaguchi MK, Alves ZCPVT. Aspectos da atividade de "notificação de barbeiros" pela população no controle de Panstrongylus megistus em 12 municípios da região noroeste do Estado de São Paulo, Brasil, 1974 a 1983. Revista de Saúde Pública 24:497-505, 1990.

6. Relatório de Oficina de Trabalho. Estratégias para vigilância epidemiológica da doença de Chagas na perspectiva da descentralização das ações. Revista da Sociedade Brasileira de Medicina Tropical 33:118-120, 1998

7. Rocha e Silva EO, Andrade JCR, Rodrigues VLC. Investigação de foco, uma das atividades das campanhas de controle dos transmissores da Tripanossomíase Americana. Revista de Saúde Pública 12:425-431, 1978.

8. Rocha e Silva EO, Guarita OF, Ishihata GK. Doença de Chagas: Atividades de controle dos transmissores no Estado de São Paulo. Revista Brasileira de Malariologia 31:99-119, 1979. 
9. Rocha e Silva EO, Rodrigues VLCC, Reyes S. Manual de normas sobre organização e funcionamento de laboratórios de diagnóstico da doença de Chagas, SUCAM, Ministério da Saúde, Brasília 1980.

10. Rocha e Silva EO, Wanderley DMV, Rodrigues VLCC. Triatoma infestans: importância, controle e eliminação da espécie no Estado de São Paulo, Brasil. Revista da Sociedade Brasileira de Medicina Tropical 31:73-88, 1998.

11. Rodrigues VLCC, Ferraz Filho AN. Distribuição e índice de infecção natural de triatomíneos capturados na região de Campinas, São Paulo, Brasil. Revista da Sociedade Brasileira de Medicina Tropical 20:25-30, 1987.

12. Rodrigues VLCC, Ferraz-Filho AN, Rocha e Silva EO, Lima VLC. Prevalência, índices de infecção e hábitos alimentares de triatomíneos. Revista da Sociedade Brasileira de Medicina Tropical 25:183-190, 1992.

13. Silva LJ. Evolução da doença de Chagas no Estado de São Paulo. Tese de Doutorado. Universidade de São Paulo. Ribeirão Preto, SP, 1981.

14. Silva RA, Bonifácio PR, Wanderley DMV. Doença de Chagas no Estado de São Paulo: comparacão entre pesquisa ativa de triatomíneos em domicílios e notificação de sua presença pela população em área sob vigilância entomológica. Revista da Sociedade Brasileira de Medicina Tropical 32:653-659, 1999.

15. Siqueira AF. Estudos sobre a reação de precipitina aplicada a identificação de sangue ingerido por triatomíneos. Revista do Instituto de Medicina Tropical 2:41-53, 1960.

16. Solís-Acosta HM, Ferreira CS, Carvalho ME. Serology of Chagas' disease in Nasca, Southern Region of Peru. Revista do Instituto de Medicina Tropical 39:107-112, 1997.

17. Souza AG, Wanderley DMV, Buralli GM, Andrade JCR. Consolidation of the control of Chagas' disease vectors in the State of Sao Paulo. Memórias do Instituto Oswaldo Cruz 79:125-131, 1984.

18. Superintendência de Controle de Endemias (SUCEN). Relatório do Grupo de Estudo do Programa de Controle da Doença de Chagas. São Paulo, 1989.

19. Wanderley DMV. Vigilância Epidemiológica da doença de Chagas no Estado de São Paulo. Revista de Saúde Pública 25:28-32, 1991.

20. Wanderley DMV. Perspectivas de Controle da doença de Chagas no Estado de São Paulo.Tese de Doutorado. Faculdade de Saúde Pública da Universidade de São Paulo. São Paulo, 1994. 